\title{
New Directions in Head and Neck Imaging
}

\author{
GAURANG V. SHAH, MD, * JEFFERY R. WESOLOWSKI, MD, SAMEER A. ANSARI, MD, \\ AND SURESH K. MUKHERJI, MD \\ Department of Radiology, University of Michigan, Ann Arbor, Michigan
}

Computerized tomography (CT) and magnetic resonance imaging (MRI), positron emission tomography (PET) and the hybrid modality of $\mathrm{PET} / \mathrm{CT}$ are sensitive and reliable tools for detection and staging of head and neck cancers. This article describes the role of PET/CT in initial staging of head and neck squamous cell carcinoma, the utility of CT/MR perfusion imaging in qualitative analysis of tumor tissue, and the usefulness of diffusion weighted MR and dynamic contrast-enhanced MR imaging in head and neck oncological imaging.

J. Surg. Oncol. 2008;97:644-648. (C) 2008 Wiley-Liss, Inc.

\section{KEY WORDS: head and neck neoplasms, diagnosis; magnetic resonance imaging; F $^{18}$-FDG PET; CT perfusion}

Diagnostic imaging has come a long way from 1895 when Conrad Roentgen discovered X-rays. Conventional radiography and ancillary techniques like fluoroscopy and tomography had a relatively minor role in evaluation of neoplasms of the head and neck unless it encroached into the aero-digestive tract. Computerized tomography (CT) was introduced in the 1970 s and revolutionized body imaging. It is inexpensive, fast, and ubiquitous in most medical centers. CT is quite good at delineating tumor extent and nodal disease. In squamous cell carcinoma, CT helped in tumor staging, which dictated patient management and related to prognosis [1]. Subsequently, helical multi-detector computerized tomography (MDCT) with 16 and now 64 detector rings has rapidly become the new industry standard in CT imaging.

Magnetic resonance imaging (MRI) in the 1980s was a quantum jump in diagnostic imaging of the human body, including imaging of head and neck pathologies. Some of earliest investigations in head and neck imaging with MRI highlighted the ability of MRI to differentiate neoplastic from inflammatory lesions. MRI provides essential information about the deep extension of clinically detected masses and also delineates additional clinically unsuspected lesions [2]. It has added value for detection of soft tissue extent, marrow involvement, and perineural spread [3]. The excellent tissue characterization and multiplanar imaging capability of MR imaging results in more accurate diagnosis of neoplastic and benign processes [4,5].

However, early investigators credited MR imaging with greater precision in head and neck imaging than was warranted [6]. Conventional MR imaging did not have the last word in histological specificity, early detection of primary malignancy and differentiating neoplastic from inflammatory lymph nodes. In spite of early enthusiasm, MR imaging did not eliminate the need for biopsies or aspirations of lesions. Spin echo imaging is still the mainstay of MR imaging, but now various new techniques hold promise for the future of head and neck imaging.

Positron emission tomography (PET) has been utilized since the 1970 s for clinical imaging. PET scanning with ${ }^{18}$ fluorodeoxyglucose (18FDG) can be used for staging and evaluation of recurrence for primary head and neck tumors. The principle for PET is based on the metabolism of the neoplasm, primary or recurrent, and is more sensitive than CT or MRI for T1-staged lesions [7]. The most recent innovation in PET systems is the hybrid PET/CT scanners. Integration of PET with CT scan in 2000 was a great leap forward and enhanced the clinical information from PET. Today all commercial PET scanners are sold as PET/CT.

Various advanced techniques are utilized for answering some of the unresolved issues from conventional CT, MRI and PET.

\section{DIFFUSION WEIGHTED MAGNETIC RESONANCE IMAGING}

Diffusion weighted imaging (DWI) with magnetic resonance relies upon the relative diffusivity of water protons within tissue. This technique is based on the amount of random (Brownian) motion that water protons undergo. During such an MRI examination, at least two short echo-planar MRI pulse sequences are applied to generate diffusion weighted images (DWI). However, some intrinsic T2 weighted information is contained in such images. Thus arithmetic processing is performed between the sets to generate apparent diffusion coefficient (ADC) maps, eliminating contributions from $\mathrm{T} 2$ signal changes. In normal tissue or in areas exhibiting vasogenic edema, the motion of water molecules is not limited and no restricted diffusion should be noted. In tissues with cytotoxic edema or in highly cellular regions, however, there is diffusion restriction, which can be measured both qualitatively and quantitatively. This technique has proven quite useful for brain imaging in differentiating between infarcted tissue and other pathological processes [8]. Early studies have also demonstrated that head and neck squamous cell cancers, likely due to their high cellular content, can demonstrate restricted diffusion [9].

While conventional imaging techniques are often adequate for demonstrating initial tumor extent, post-treatment changes can be difficult to delineate from small volumes of tumor recurrence, as both may enhance in a similar fashion. Diffusion weighted MRI can aid in these situations. Inflammatory or post-treatment tissue does not

*Correspondence to: Gaurang V. Shah, MD, Department of Radiology, UMHS, B2A209, 1500 E Medical Center Drive, Ann Arbor, MI 48109. Fax: 734-764-2412. E-mail: gvshah@med.umich.edu

Received 6 February 2008; Accepted 12 February 2008

DOI 10.1002/jso.21022

Published online in Wiley InterScience

(www.interscience.wiley.com).

(C) 2008 Wiley-Liss, Inc. 
demonstrate restricted diffusion, likely due to relative low cellularity. Recurrent or residual disease, however, contains regions of increased cellularity and thus should demonstrate restricted diffusion (Fig. 1) [10]. Recent studies have maintained that such imaging can be a useful adjunct in assessing post-treatment disease and may aid in diagnosis, particularly with regard to tissue sampling [11]. Diffusion weighted imaging of the orophayrynx can easily be performed at the time of MR conventional imaging and adds approximately only 1-2 min of additional time to the examination. High sensitivities and specificities better than CT or conventional MRI are also reported in staging of neck lymph nodes in squamous cell carcinoma $[12,13]$. Whole body DWI at high b-values with ADC mapping is technically feasible and improves assessment of metastatic spread in routine MR examinations [14]. However, technical standardization is still not achieved; results obtained depend on selection of b-values. Magnetic field inhomogeneity and suboptimal placement of receiver coils can have a negative impact on image quality [10]. Interpretation of DWI in the head and neck requires training and experience and more research is still needed in this field.

\section{PERFUSION IMAGING}

Perfusion imaging, whether performed with CT or MRI, evaluates dynamic microscopic blood flow changes through a region of interest. Changes in tissue signal intensity (MRI) or attenuation (CT) are measured during a dynamic contrast infusion. Blood flow, blood volume, and transit time parameters of tissue regions can be then generated, either on the CT scanner or on a separate workstation with commercially available software. Perfusion characteristics of tissue demonstrate changes in blood flow or volume of the interrogated areas depending on the underlying pathologic processes. This technique has been previously studied in characterizing brain ischemia, particularly in identifying infarcted tissue versus tissue at risk [15]. Neoplastic tissue also demonstrates changes in perfusion characteristics. Early studies have shown that oropharyngeal tumor tissue demonstrates increased blood volume and blood flow with decreased transit times in comparison to normal tissue [16].

Generally, these findings may not add substantial additional information regarding tumor extent at the time of diagnosis. However, such imaging may be of benefit in qualitative analysis of tumor tissue. Specifically, additional recent studies have demonstrated that squamous cell carcinomas of the upper aerodigestive tract with increased blood volume/flow are more chemosensitive than other lesions with relative decreased perfusion parameters (Fig. 2). This is likely due to relative increased oxygenation and metabolism of such lesions [17]. Such perfusion techniques could be particularly useful in determining which patients would benefit from such medical treatment, as opposed to surgical therapies which may not always preserve organ function.

An additional area of interest is in regard to tumor recurrence or regression. Again, conventional MRI or CT may simply demonstrate increased contrast enhancement within the treated neck. Morphologic changes in tissue appearance (such as increase in size or nodularity) may not be well demonstrated on early post-treatment conventional imaging. However, recent studies have concluded that for recurrent oral cavity and oropharyngeal carcinomas, perfusion parameters are altered. Specifically, blood volume and blood flow within recurrent tumor tissue are elevated in comparison to therapy-altered tissue, with corresponding decreases in transit time [18]. Perfusion imaging, like diffusion imaging, adds little time to either conventional MRI or CT examinations and can also be obtained noninvasively [19]. Blood flow, volume, and transit time maps can be generated either on the unit or on a separate workstation.

\section{PET CT FOR DIAGNOSIS AND INITIAL STAGING}

The clinical usefulness and role of FDG-PET CT for detection of lymph node involvement and recurrences in patients with head and neck cancer is very well-established [20]. It has been found to be superior to conventional imaging work-ups in the evaluation of patients with head and neck malignancies [21]. FDG PET is also found to be more accurate than CT/MRI imaging in oral cavity cancer [22]. However, potential clinical applications include pretreatment staging, treatment monitoring and evaluation of the previously treated patients [23]. The current practice is not in favor of utilizing CT-PET for staging of all newly diagnosed squamous cell carcinomas. However, PET can detect metastatic cervical lymph nodes, which may be clinically occult and may not be detected by CT or MR imaging [24]. It can also detect primary head and neck squamous cell carcinomas greater than $1 \mathrm{~cm}$ in size.

PET-CT may be performed in squamous cell carcinoma to evaluate for possible occult distant metastases to the lungs or bones [25]. The presence of pulmonary metastases upstages a patient from M0 to M1 and alters the treatment regimen (Fig. 3). Routine imaging work-up for the patient with pulmonary squamous cell carcinoma includes conventional radiography of the chest at most institutions. Chest CT is performed in patients with advanced stage disease. A solitary nodule on CT scan may represent a metastasis or a granuloma. PET would be
A

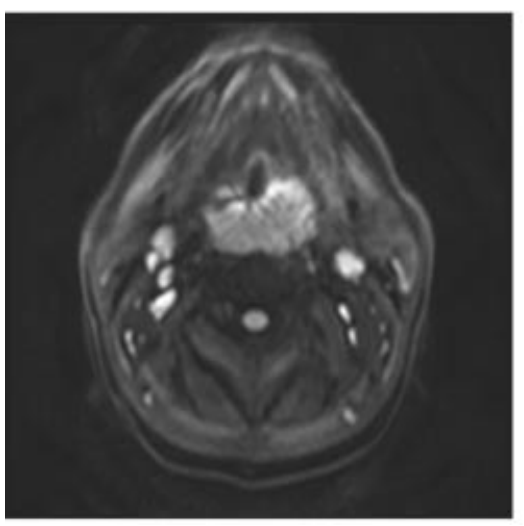

B

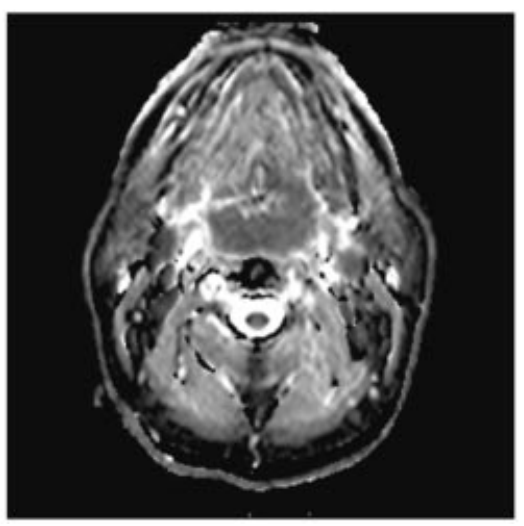

C

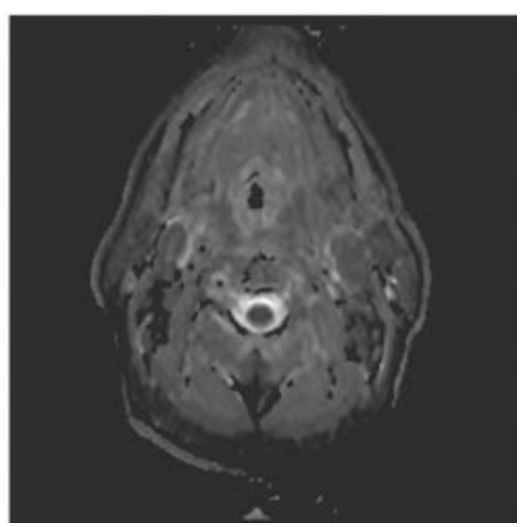

Fig. 1. Large posterior oropharyngeal wall squamous cell carcinoma demonstrates increased DWI (A) and decreased ADC (B) signal intensity at presentation. Post-therapy, the lesion has decreased greatly in size (C). 


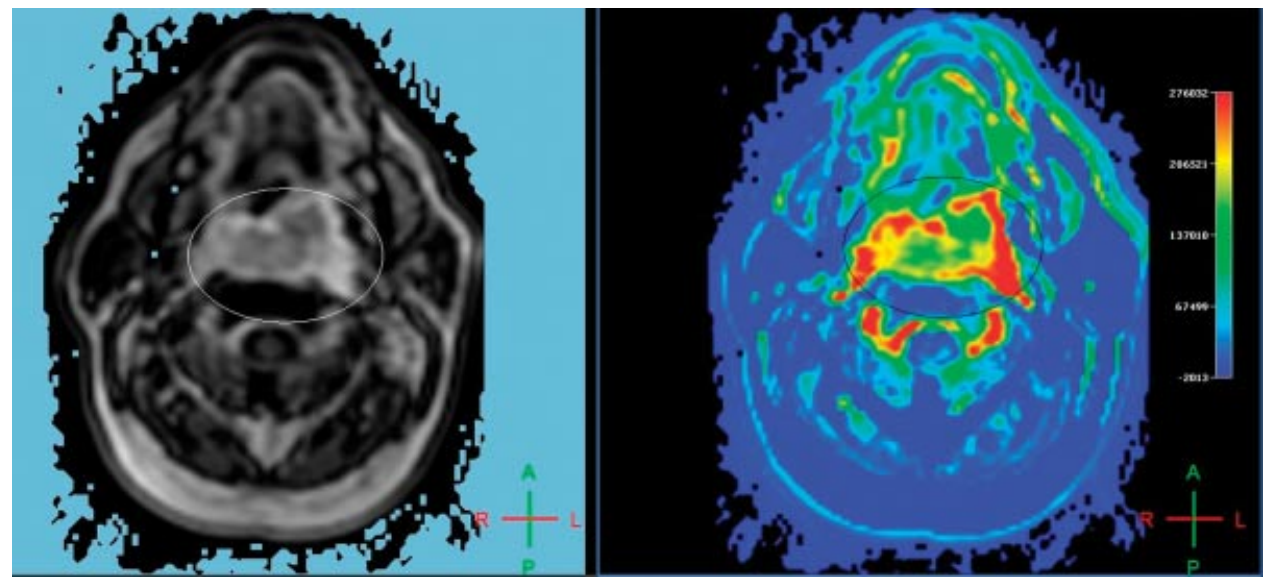

Fig. 2. Blood volume map of the same patient as in Figure 1A-C demonstrates increased perfusion values of the lesion (circled) in comparison to the adjacent tissues at presentation. [Color figure can be viewed in the online issue, available at www.interscience.wiley.com.]

helpful in this evaluation as an FDG-positive nodule would likely be metastatic and may require biopsy. An FDG-negative nodule may likely indicate a granuloma. Various studies have evaluated the diagnostic accuracy of PET-FDG for detecting metastatic cervical lymph nodes. It has a high negative predictive value (NPV) of approximately $90 \%$, which is more than any other imaging modality. With future technological advances, PET-CT is likely to have high resolution CT imaging. This is likely to increase the diagnostic accuracy of combined PET-CT, which may have greater impact on management of NO [26] disease.

The ability of PET-CT to detect unknown primary tumors of the upper arodigestive tract is well-documented [27-29]. PET can detect squamous cell carcinoma in $30-50 \%$ of patients presenting with an unknown primary tumor. PET-CT is generally performed after confirming the presence of metastatic squamous cell carcinoma. It is usually performed before endoscopic biopsies to improve the tissue yield. This diagnostic yield can increase with PET-CT because as it improves the anatomic localization of areas of abnormal FDG uptake $[30,31]$.

A

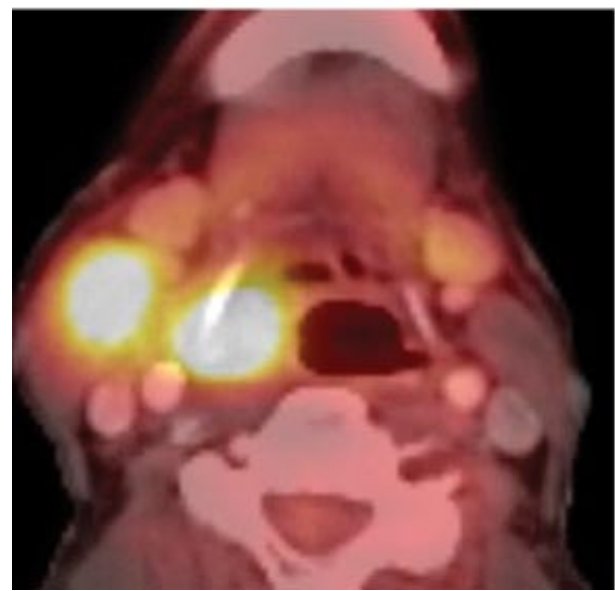

PET-CT can also be potentially utilized for determining response to chemotherapy and/or radiation. Comparison of pretreatment standard uptake values (SUVs) to SUVs 2 weeks into treatment can allow measurement of the speed of response and also the sensitivity of the tumor to the treatment technique [32]. Poorly responsive tumors can then be treated to higher effective tumor doses of radiation, or surgery can be performed. Initial results suggest that PET-CT can be used to assist in defining primary site and nodal tumor targets for radiation therapy approaches. PET-CT is a useful adjuvant to clinical staging of squamous cell carcinoma and its utilization will increase with advancement of technology.

\section{DYNAMIC CONTRAST-ENHANCED MR IMAGING}

Enhancement of a body tissue depends on vascularity, capillary permeability, renal clearance and volume and composition of extracellular fluid [33,34]. After intravenous administration of a bolus
B

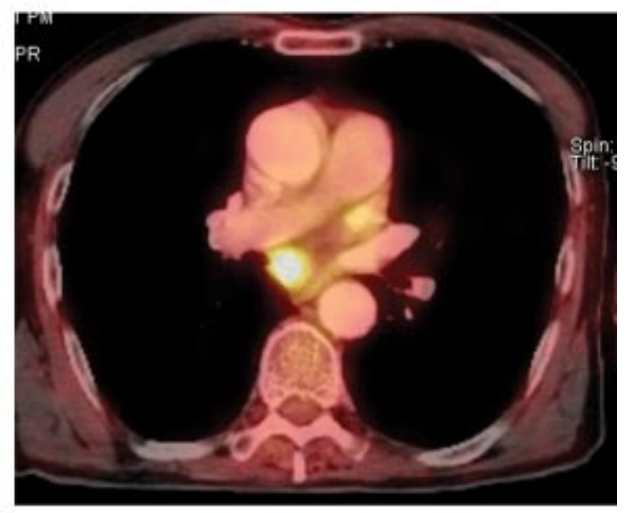

Fig. 3. T3N2 pyriform sinus carcinoma. A: Axial PET-CT demonstrates avid FDG uptake in a right pyriform sinus carcinoma and a metastatic right cervical lymph node. B: PET-CT of the chest shows a mediastinal mass with focal increased uptake. This was not detected on conventional radiography of the chest. [Color figure can be viewed in the online issue, available at www.interscience.wiley.com.] 
A

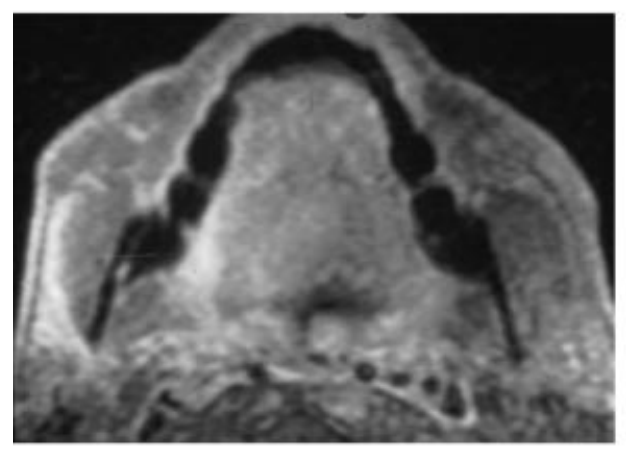

B

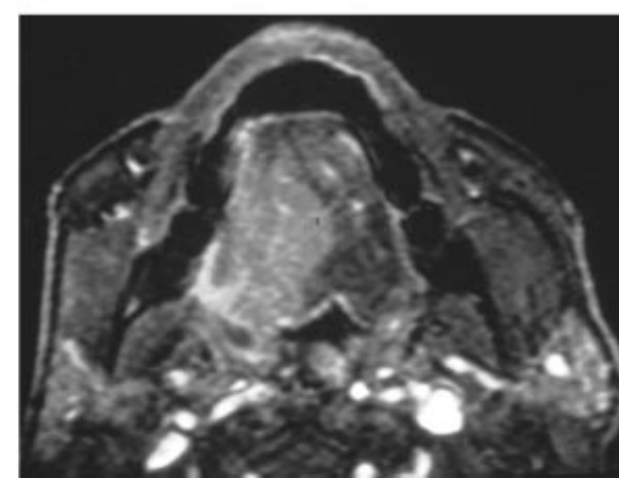

Fig. 4. Forty-five-year-old with carcinoma of tongue. A: Post-contrast T1-weighted image exhibits an irregular soft tissue mass with indistinct margins, patchy enhancement at the base of the tongue on the right side. B: Dynamic contrast-enhanced MR with time-of-flight spoiled gradient-recalled imaging exhibits intense post-contrast enhancement with the real extent of the tumor and further anterior extension of the mass than seen on conventional post-contrast imaging.

of paramagnetic contrast agent, it rapidly passes in to extravascular space except in brain, retina and testes where specific vascular barriers prevent it. This passage depends on the flow of the blood, and the surface area and thickness of micro vessels. In case of malignant tumors and lymph nodes, the vascular permeability is greater than inflow of blood and hence blood perfusion is dominant factor for contrast enhancement [35]. The tumor microenvironment has increased microvascular permeability and diameter, increased flow and blood volume of microvasculature, increased tissue oxygenation and metabolism, and many times increased fractional volume of extravascular extracellular space [36]. All these parameters result in the rapid onset of contrast enhancement, which may taper off rapidly or may persist depending on tissue parameters (Fig. 4A,B). After passing in to extracellular spaces, the contrast begins to diffuse into tissue compartments farther than vasculature and eventually over several minutes to hours, diffuses back into vasculature. In areas of fibrosis and necrosis, the elimination of contrast is slower due to slower exchange rates and hence they exhibit persistent delayed contrast characteristics [37]. Tissue perfusion and blood volume can be evaluated and measured with a designed protocol of T1- and sometimes $\mathrm{T} 2 *$-weighted images. Field inhomogeneities caused by intravascular contrast media can be quantified by the $\mathrm{T} 2 *$-weighted method [38] while shortening of T1 relaxation times can be quantified by $\mathrm{T} 1$-weighted sequences.

Dynamic contrast-enhanced MR imaging has been utilized to study head and neck cancers [39-43]. Escott et al. [39] did not analyze signal intensity versus time curves, but noted that dynamic gradientecho MR imaging was superior to conventional contrast-enhanced spin-echo imaging in delineating margins and extent of tumor. Guckel et al. [42] used dynamic contrast-enhanced MR imaging to evaluate signal intensity versus time for squamous cell carcinoma of the oral cavity and oropharynx and found that the time/intensity curves of the tumors could be divided into two groups, one showing a rapid enhancement pattern with an early peak and then a continuous decrease and one showing a slower but continuous increase that then gradually decreased. Baba et al. [41] found that dynamic MR imaging was useful in distinguishing persistent head and neck tumor from post -radiation fibrosis by showing early enhancement in residual tumor. Hoskin et al. [43] also assessed dynamic contrast-enhanced MR imaging as a predictor of response to accelerated radiation therapy for advanced head and neck cancer. They found that tumors with diminished tumor perfusion at the end of radiotherapy are more likely to respond to radiation and those that show greater post-contrast enhancement after accelerated radiotherapy are likely to fail locally and suggested that tumor blood flow is an important parameter in predicting the outcome from radiotherapy. However, it was felt that dynamic contrast-enhanced imaging has a very limited and non-significant role and that unless invasion or T2 hypointensity was present, dynamic MR imaging has a relatively small role to play in evaluating nonvascular masses of head and neck [44].

\section{REFERENCES}

1. Gatenby RA, Mulhern CB, Jr., Strawitz J, et al.: Comparison of clinical and computed tomographic staging of head and neck tumors. Am J Neuroradiol 1985;6:399-401.

2. Stark DD, Moss AA, Gamsu G, et al.: Magnetic resonance imaging of the neck. Part II. Pathologic findings. Radiology 1984; 150:455-461.

3. Weber AL, Romo L, Hashmi S: Malignant tumors of the oral cavity and oropharynx: Clinical, pathologic and radiologic evaluation. Neuroimag Clin N Am 2003;13:443-464.

4. Wippold FJ: Head and Neck Imaging: The role of CT and MRI J Magn Reson Imaging 2007;25:453-465.

5. McCabe KJ, Rubinstein D: Advances in head and neck imaging. Otolaryngologic Clin N Am 2005;38:307-319.

6. Yousem DM: Dashed hopes for MR imaging of the head and neck: The power of the needle. Radiology 1992;184:25-26.

7. Laubenbacher C, Saumweber D, Wagner-Manslau C: Comparison of fluorine-18-fluorodeoxyglucose PET, MRI and endoscopy for staging head and neck squamous-cell carcinomas. J Nucl Med 1992;36:1747-1757.

8. Schaefer PW, Copen WA, Lev MH, et al.: Diffusion-weighted imaging in acute stroke. Magn Reson Imaging Clin N Am 2006; 14:141-168.

9. Wang J, Takashima S, Takayama F, et al.: Head and neck lesions: Characterization with diffusion-weighted echo-planar MR imaging. Radiology 2001;220:621-630.

10. Hermans R, Vandecaveye V: Diffusion-weighted MRI in head and neck cancer. Cancer Imaging 2007;7:126-127.

11. Abdel Razek AA, Kandeel AY, Soliman N, et al.: Role of diffusion-weighted echo-planar MR imaging in differentiation of residual or recurrent head and neck tumors and posttreatment changes. Am J Neuroradiol 2007;28:1146-1152.

12. Abdel Razek AA, Soliman NY, Elkhamary S, et al.: Role of diffusion weighted MR imaging in cervical lymphadenopathy. Eur Radiol 2006;16:1468-1477.

13. Sumi M, Sakihama N, Sumi T, et al.: Discrimination of metastatic cervical lymph nodes with diffusion-weighted MR imaging in patients with head and neck cancer. Am J Neuroradiol 2003;79: 681-687. 
14. Lichy MP, Aschoff P, Plathow C, et al.: Tumor detection by diffusion-weighted MRI and ACD-mapping-initial clinical experiences in comparison to PET-CT. Invest Radiol 2007;42:605613.

15. Schaefer PW, Ozsunar Y, He J, et al.: Assessing tissue viability with MR diffusion and perfusion imaging. Am J Neuroradiol 2003;24:436-443.

16. Gandhi D, Hoeffner EG, Carlos RC, et al.: Computed tomography perfusion of squamous cell carcinoma of the upper aerodigestive tract. Initial results. J Comput Assist Tomogr 2003;27:687-693.

17. Zima A, Carlos R, Gandhi D, et al.: Can pretreatment CT perfusion predict response of advanced squamous cell carcinoma of the upper aerodigestive tract treated with induction chemotherapy? Am J Neuroradiol 2007;28:328-334.

18. Bisdas S, Baghi M, Smolarz A, et al.: Quantitative measurements of perfusion and permeability of oropharyngeal and oral cavity cancer, recurrent disease, and associated lymph nodes using firstpass contrast-enhanced computed tomography studies. Invest Radiol 2007;42:172-179.

19. Schmitt P, Kotas M, Tobermann A, et al.: Quantitative tissue perfusion measurements in head and neck carcinoma patients before and during radiation therapy with a non-invasive MR imaging spin-labeling technique. Radiother Oncol 2003;67:2734.

20. Paulus P, Sambon A, Vivegnis D, et al.: 18FDG-PET for the assessment of primary head and neck tumors: Clinical, computed tomography, and histopathological correlation in 38 patients. Laryngoscope 1998;108:1578-1583.

21. Schmid DT, Stoeckli SJ, Bandhauer F, et al.: Impact of positron emission tomography on the initial staging and therapy in locoregional advanced squamous cell carcinoma of the head and neck. Laryngoscope 2003;113:888-891.

22. Ng SH, Yen TC, Chang JT, et al.: Prospective study of [18F]fluorodeoxyglucose positron emission tomography and computed tomography and magnetic resonance imaging in oral cavity squamous cell carcinoma with palpably negative neck. J Clin Oncol 2006;24:4371-4376.

23. Mukherji SK, Bradford CR: Controversies: Is there a role for positron-emission tomographic $\mathrm{CT}$ in the initial staging of head and neck squamous cell carcinoma? Am J Neuroradiol 2006;27: 243-245.

24. Di Martino E, Nowak B, Hassan HA, et al.: Diagnosis and staging of head and neck cancer: A comparison of modern imaging modalities (positron emission tomography, computed tomography, color-coded duplex sonography) with panendoscopic and histopathologic findings. Arch Otolaryngol Head Neck Surg 2000;126:1457-1461.

25. Benchaou M, Lehmann W, Slosman DO, et al.: The role of FDGPET in the preoperative assessment of $\mathrm{N}$-staging in head and neck cancer. Acta Otolaryngol 1996;116:332-335.

26. Schwartz DL, Rajendran J, Yueh B, et al.: FDG-PET prediction of head and neck squamous cell cancer outcomes. Arch Otolaryngol Head Neck Surg 2004;130:1361-1367.

27. Mukherji SK, Drane WB, Mancuso AA, et al.: Occult primary tumors of the head and neck: Detection with 2-(F-18) fluoro-2deoxy-D-glucose SPECT. Radiology 1996;199:761-766.
28. Kole AC, Nieweg OE, Pruim J, et al.: Detection of unknown occult primary tumors using positron emission tomography. Cancer 1998;82:1160-1166.

29. AAssar OS, Fischbein NJ, Caputo GR, et al.: Metastatic head and neck cancer: Role and usefulness of FDG PET in locating occult primary tumors. Radiology 1999;210:177-181.

30. Sheikholeslam-Zadeh R, Choufani G, Goldman S, et al. Unknown primary detected by FDG-PET: A review of the present indications of FDG-PET in head and neck cancers. Acta Otorhinolaryngol Belg 2002;56:77-82.

31. Gutzeit A, Antoch G, Kuhl H, et al.: Unknown primary tumors: Detection with dual-modality PET/CT: Initial experience. Radiology 2005;234:227-234.

32. Lowe VJ, Dunphy FR, Varvares M, et al.: Evaluation of chemotherapy response in patients with advanced head and neck cancer using [F-18]fluorodeoxyglucose positron emission tomography. Head Neck 1997;19:666-674.

33. Brasch RC: New directions in the development of MR imaging contrast media. Radiology 1992;183:1-11.

34. Kormano M, Dean PB: Extravascular contrast material: The major component of contrast enhancement. Radiology 1976;121: $379-382$.

35. Taylor JS, Tofts PS, Port R, et al.: MR imaging of tumor microcirculation: Promise for the new millennium. J Magn Reson Imaging 1999;10:903-907.

36. Padhani AR: Dynamic contrast-enhanced MRI in clinical oncology: Current status and future directions. J Magn Reson Imaging 2002;16:407-422.

37. Matsubayashi R, Matsuo Y, Edakuni G, et al.: Breast masses with peripheral rim enhancement on dynamic contrast-enhanced MR images: Correlation of MR findings with histologic features and expression of growth factors. Radiology 2000;217:841-848.

38. Sorensen AG, Tievsky AL, Ostergaard L, et al.: Contrast agents in functional MR imaging. J Magn Reson Imaging 1997;7:4755.

39. Escott EJ, Rao VM, Ko WD, et al.: Comparison of dynamic contrast-enhanced gradient-echo and spin-echo sequences in MR of head and neck neoplasms. Am J Neuroradiol 1997;18:14111419.

40. Baba Y, Yamashita Y, Onomichi M, et al.: Dynamic magnetic resonance imaging of head and neck lesions. Top Magn Reson Imaging 1999;10:125-129.

41. Baba Y, Furusawa M, Murakami R, et al.: Role of dynamic MRI in the evaluation of head and neck cancers treated with radiation therapy. Int J Radiat Oncol Biol Phys 1997;37:783-787.

42. Guckel C, Schnabel K, Deimling M, et al.: Dynamic snapshot gradient-echo imaging of head and neck malignancies: Time dependency and quality of contrast-to-noise ratio. MAGMA 1996;4:61-69.

43. Hoskin PJ, Saunders MI, Goodchild K, et al.: Dynamic contrast enhanced magnetic resonance scanning as a predictor of response to accelerated radiotherapy for advanced head and neck cancer. $\mathrm{Br}$ J Radiol 1999;72:1093-1098.

44. Yousem DM: Dynamic MR imaging of the head and neck: An idea whose time has come... and gone? Radiology 1993;189: $659-660$ 